Running head: PERITRAUMATIC REACTIONS, GENDER AND ASD

\title{
Gender Differences in the Prediction of Acute Stress Disorder from Peritraumatic Dissociation and Distress among Victims of Violent Crimes
}

\author{
Julie Boisclair Demarble, Christophe Fortin, Bianca D'Antono and Stéphane Guay \\ Université de Montréal, Montréal, Québec, Canada
}

\section{Author note}

Julie Boisclair Demarble, Department of Psychology, Université de Montréal; Christophe Fortin, School of Criminology, Université de Montreal, and' Trauma Studies Center, Institut Universitaire en Santé Mentale de Montréal, Montreal, Quebec, Canada; Bianca D'Antono, Department of Psychology, Université de Montréal, and Research Center, Montreal Heart Institute, Montreal, Quebec, Canada; Stéphane Guay, School of Criminology, Université de Montreal and Trauma Studies Center, Institut Universitaire en Santé Mentale de Montréal.

This research was supported by grants awarded to Dr Guay by the Canadian Institutes of Health and Research (CIHR). Julie Boisclair Demarble was supported by a Masters' scholarship from the Fonds de la recherche en santé du Québec (FRSQ). We would also like to thank the Quebec's Crime Victim's Assistance Centers of Montreal and the Institut Universitaire en Santé Mentale de Montréal for their generous support of this study.

Correspondence concerning this article should be addressed to Julie Boisclair Demarble, Department of Psychology, Université of Montréal, Montreal, Québec, Canada.

Email: julie.boisclair.demarble@umontreal.ca 


\title{
Gender Differences in the Prediction of Acute Stress Disorder from Peritraumatic Dissociation and Distress among Victims of Violent Crimes
}

\begin{abstract}
Background. Peritraumatic dissociation and distress are strong predictors of Acute Stress Disorder (ASD) and Posttraumatic Stress Disorder (PTSD) development. However, there is limited data concerning gender differences in these relations, particularly among victims of violent crimes (VVC). Objective. Examine whether peritraumatic dissociation and distress predict the number of ASD symptoms differently for men and women VVC. Methods. 162 adults (97 women, $\mathrm{M}_{\mathrm{age}}=39.6 \mathrm{yrs}$.), $63 \%$ of whom experienced physical assaults, completed the Acute Stress Disorder Interview, the Peritraumatic Dissociative Experience Questionnaire, and the Peritraumatic Distress Inventory. Analyses included t-tests and multiple hierarchical regressions models controlling for known PTSD risk factors. Results. The regression model showed dissociation and distress to be significant predictors of ASD for both men and women ( $\beta$ $=.349$ and $\beta=.312$ respectively; all $\mathrm{p} \leq .001)$. A significant 3 -way interaction was also observed between peritraumatic distress (PDI), past potentially traumatic experiences and gender. In simple slopes analyses, the combination of high levels of PDI and of a high number of past potentially traumatic events were associated with greater risk of ASD in men only $(b=3.78$, $\mathrm{p}<.001)$. However, women experienced greater PDI $(\mathrm{t}(157)=5.844, \mathrm{p}=.005)$ than men, and elevated distress was associated with more ASD symptoms independently of past traumatic events. Major implications. Gender differences were revealed as a function of past potentially traumatic experiences. There is a cumulative impact of past potential traumas and current distress that predicts ASD in men, while in women; it contributes to ASD via increased distress.
\end{abstract}


Keywords: victims of violent crimes; acute stress disorder; peritraumatic dissociation; peritraumatic distress; gender 


\section{Gender Differences in the Prediction of Acute Stress Disorder from Peritraumatic Dissociation and Distress among Victims of Violent Crimes}

\section{Introduction}

Interpersonal violence is the most common traumatic event in the general population (Breslau et al., 1998; Norris \& Kaniasty, 1994; Stein, Walker, Hazen, \& Forde, 1997) and is associated with a higher probability of subsequent posttraumatic stress disorder (PTSD), compared to other trauma types (Breslau, 2001; Bryant \& Harvey, 2003; Kessler, 2000; Van Ameringen, Mancini, Patterson, \& Boyle, 2008). In addition, more severe and chronic symptoms of PTSD have been reported for victims of violent crimes (VVC) (Breslau et al., 1998; Norris \& Kaniasty, 1994; Stein et al., 1997). The interpersonal nature of the traumatic event may contribute to greater psychological distress.

While less is known about the acute stress and peritraumatic reactions of this population, investigations showed that more VVC meet criteria for an acute stress disorder, (20-50\%) compared to motor vehicle accident (MVA) survivors and burn patients (13-20\% and 23.6\%, respectively) (Bryant \& Harvey, 2003; McKibben, Bresnick, Askay, \& Fauerbach, 2008). More specifically, in a study on victims of interpersonal violence, $24 \%$ met criteria for a full ASD diagnosis and an additional 21\% obtained a subclinical ASD (Elklit \& Brink, 2004). Similar prevalence rates were also found among armed robbery victims (25\%)(Elklit, 2002). More recently, in a study with female victims of sexual assault, the rate of ASD reached 59\% (Elklit \& Christiansen, 2010).

Acute pathological reactions to trauma were specifically addressed by the introduction of the acute stress disorder (ASD) diagnosis in DSM-IV(American Psychiatric Association, 2000). It acknowledged the fairly common dissociative symptomatology experienced during or immediately after a traumatic event (Cardeña \& Carlson 2011). In this sense, ASD shares many 
symptoms with PTSD, but requires more dissociative responses (at least three) and can only be diagnosed up to one month following the traumatic event. In DSM-5, acute stress responses are not divided into clusters and the presence of specific dissociative symptoms is no longer required (American Psychiatric Association, 2013). Alternately, ASD is conceptualized in terms of severity, requiring the presence of 9 symptoms (out of 14), irrespective of symptoms categories (Bryant, Friedman, Spiegel, Ursano, \& Strain, 2011). Nonetheless, dissociative reactions are still considered in two ways; first, by the addition of other dissociative symptoms to the ASD classification (Armour et al., 2015), and second, by the inclusion of a dissociative subtype within the PTSD diagnosis.

Peritraumatic dissociation (PD) refers to alterations, disruptions or gaps in awareness and memory that occur during the traumatic event or immediately afterward (Bryant, 2007). It can include manifestations of derealization (e.g.,, distorted perception of reality), depersonalization (e.g., feeling disconnected from one's own body), dissociative amnesia, emotional numbing or detachment and reduced awareness of one's surroundings. During a traumatic event, PD may serve a protective function as it occludes the distressing event from consciousness (Bryant, 2006) by allowing one to detach one's self emotionally (Feeny, Zoellner, \& Foa, 2000). It can also be viewed as psychologically adaptive as the individual is momentarily distancing himself from the aversive experience. Unfortunately, not properly coping with distress could also maintain it (Cardeña \& Carlson 2011). Dissociative reactions often prevent proper encoding of the event and prevent emotional processing of trauma memories (Bryant, 2007; Friedman, Keane, \& Resick, 2007). As found in a recent study, PD may be more likely to occur in very severe and intolerable events, such as interpersonal violence (Hetzel-Riggin \& Roby, 2013). Moreover, high prevalence 
rates $(73 \%)$ were observed for the ASD dissociative criteria in a sample of robbery victims and victims of physical assaults (Elklit, 2002).

Peritraumatic distress (PDI) is a construct comprised of emotional and physical stress responses, including trembling and palpitations, which occur during or immediately after the traumatic event. Negative emotions include fear, horror, helplessness, shame and anger, as well as the perception that one's life was endangered. The more one perceives a threat to life, the greater the severity of symptoms across all three clusters of ASD (i.e., intrusion, avoidance and hyper vigilance) (Elklit, 2002; Norris \& Kaniasty, 1994; Johansen, Wahl, Eilertsen, Hanestad, \& Weisaeth, 2006). It is also hypothesized that presenting with high levels of negative emotions during a traumatic event mobilizes available resources that would otherwise facilitate problem solving. This could increase the risk that the event will be negatively appraised, and of development of subsequent PTSD (Olff, Langeland, Draijer, \& Gersons, 2007).

Both peritraumatic dissociation and distress reactions may increase risk for ASD and PTSD (Breh \& Seidler, 2007; Elklit \& Brink, 2004; Hansen \& Elklit, 2011; Johansen et al., 2006; Martin \& Marchand, 2003), with associations showing moderate effect sizes (Brewin, Andrews, \& Valentine, 2000a; Ozer, Best, Lipsey, \& Weiss, 2003). Effect sizes for PDI were greater among crime victims compared to accident survivors (Norris \& Kaniasty, 1994; Ozer et al., 2003). Moreover, presenting with intense peritraumatic emotional distress predicted ASD in victims of a bank robbery (Hansen \& Elklit, 2011). While not consistently observed (Marshall \& Schell, 2002; van der Velden \& Wittmann, 2008), peritraumatic dissociation was found to predict ASD severity in survivors of MVA and physical assaults (Panasetis \& Bryant, 2003). Contradictory evidence regarding the predictive power of peritraumatic reactions for ASD and PTSD could be explained by the confounding effects of other known risk factors(Bryant, 2007; 
Bryant et al., 2011) such as experience of childhood trauma or multiple trauma histories (HetzelRiggin \& Roby, 2013). Differences in research designs (cross-sectional vs. prospective vs. retrospective) may also have contributed to mixed findings. Retrospective assessments completed a few months after the trauma often reveal higher levels of PD compared to assessments soon after the time of the trauma (Ozer et al., 2003). In addition, assessment of ASD in the current literature rarely involved structured clinical interviews. Moreover, ASD has sometimes been assessed using measures of PTSD, which raises concerns regarding reliability, sensitivity and specificity of the diagnosis. Finally, gender is likely to influence peritraumatic reactions, but research including gender as a moderator is sparse.

Epidemiological studies also report that females are usually more likely to experience extreme fear then males (Kimerling, Ouimette \& Wolfe, 2002). Among victims of interpersonal violence specifically, women tend to present with more ASD symptoms then men (Bryant \& Harvey, 2003; Elklit, 2002; Panasetis \& Bryant, 2003) and show a greater risk of developing PTSD (Creamer, Burgess, Buckingham, \& Pattison, 1993; Tolin \& Foa, 2006). Men and women show differences in their risk for exposure to traumatic events and in their lifetime prevalence rates for PTSD (Breslau, Chilcoat, Kessler, Peterson, \& Lucia, 1999; Tolin \& Foa, 2006; Van Ameringen et al., 2008). Some studies have also reported gender differences in the intensity of peritraumatic reactions. Higher levels of PDI were found among female police officers in comparison to their male counterparts (Brunet et al., 2001) and elevated levels of peritraumatic dissociation and distress were observed among women victims of physical assaults compared to men (Johansen et al., 2006). Similar results highlighting men's resilience to trauma were recently obtained among college students $(\mathrm{N}=61)$ victims of interpersonal violence (Hetzel-Riggin \& Roby, 2013), where greater PD levels were observed among women. Intense trauma-related 
emotions, particularly fear and horror, were experienced more frequently by women (Brewin, Andrews, \& Rose, 2000b). Therefore, gender needs to be taken into consideration when studying peritraumatic reactions and their effects on prediction of ASD.

Prospective research that has investigated gender differences in the prediction of ASD specifically remains scarce. Studies on MVA victims found that dissociative reactions were a better predictor for ASD among women (Fullerton \& al. 2001) and this seemed to explain in part women's higher risk for PTSD in another study (Irish et al., 2011). Moreover, prior exposure and multiple trauma histories may also play a role in the observed gender differences. To our knowledge this was never examined in VVC. This highlights the importance of considering peritraumatic reactions and prior exposure to further our understanding of gender differences in ASD development.

The current study aimed to analyze gender-based differences among victims of violent crimes. The purpose was twofold: first, we sought to examine gender differences in levels of peritraumatic dissociation and distress and whether gender had an impact in the prediction of ASD symptoms from peritraumatic reactions. Second, we further investigated interactive effects of pertitraumatic reactions with gender and past potential traumatic events on the prediction of ASD. Considering the exceptionally severe nature of the events to which VVC are exposed to, and based on previous results across various trauma populations (Elklit, 2002; Grieger, Fullerton, \& Ursano, 2003; Johansen et al., 2006; Olff et al., 2007), it was hypothesized that high levels of PD and PDI would be observed in the overall sample (Perry, Pollard, Blakley, Baker, \& Vigilante, 1995; Steuwe, Lanius, \& Frewen, 2012). Keeping in mind the studies that have showed women's greater risk, the major hypothesis of this study was that peritraumatic reactions would be more intense among women participants and that these would be stronger predictors of 
ASD in women compared to men. No hypotheses were developed for the second objective, as this research question was more exploratory in nature.

\section{Participants}

\section{Methods}

Victims of violent crimes were recruited within a larger longitudinal study on the risk for ASD and PTSD, and the efficacy of a cognitive-behavioral treatment involving a significant other in the treatment of ASD. A sample of 89 men and 125 women, aged 18-75 years $\left(\mathrm{M}_{\mathrm{age}}=\right.$ 39.4 years) was recruited via collaborations with the Montreal Crime Assistance Center (CAC) from 2009-2014. To be eligible, participants had to meet the following criteria: (a) having been exposed to a violent crime within thirty days prior to the reference from the CAC; (b) be at least 18 years of age (c) have a good mastery of French or English. Exclusion occurred if they presented with a current psychiatric diagnosis, bipolar disorder, past or present psychotic episode, organic brain disorder, traumatic brain injury and/or active suicidal ideations.

\section{Procedure}

A CACs' counselor initially informed each participant of the research project. Individuals willing to participate were all contacted within 28 days following exposure to the traumatic event by telephone and received a standardized and detailed description of the study. Following verbal consent, a short interview (20 min.) assessed the victim's eligibility to the project and specified the event's circumstances.

Eligible participants then underwent semi-structured clinical interviews at the Trauma Studies Center a mean of 24.6 days $(\mathrm{SD}=9.68)$ after the event. Signed consent was obtained. Trained doctoral level female research assistants conducted interviews. The interview assessed the presence of an ASD, peritraumatic distress and dissociation, as well as other risk factors for 
ASD and PTSD. Socio demographic questionnaires were administered and participants were again asked to provide a detailed description of the traumatic event. For each completed assessment, participants received a $20 \$$ compensation. All interviews were audiotaped in order to evaluate inter-rater reliability. The Research and Ethics Board of the Institut Universitaire en Santé Mentale de Montréal approved this study.

\section{Measurement}

\section{Socio-demographic variables}

Data on gender, age in years, marital and employment status, and education levels were collected.

\section{Psychological Variables}

Only variables of relevance for the present article are reported below. ASD was assessed using the semi-structured Acute Stress Disorder Interview (ASDI;(Bryant, Harvey, Dang, Sackville, \& Basten, 1998)). As data collection for the current study was completed before publication of the DSM-5, presence of ASD was established using criteria listed in the DSM-4-TR. The ASDI assesses ASD criteria according to the presence of 19 symptoms (A1; perception of life threat, A2 presence of fear, horror and/or helplessness, B; at least 3 dissociative symptoms, C; at least 1 re-experiencing symptom, D; at least 1 avoidance symptom, E; at least 1 hyperarousal symptom) giving a total acute stress disorder severity score. Psychometric properties are excellent (0.90 for internal consistency and test-retest reliability 0.88). Sensitivity and specificity are also very good (91\% and $93 \%$ respectively) (Harvey \& Bryant, 1999). ASD was evaluated as a continuous variable measuring the severity of the diagnosis based on the number of symptoms met. This allowed for inclusion of participants with a subclinical disorder. Total severity scores for ASD have been found to be better predictors of 
ASD than meeting each individual symptom cluster (Hansen \& Elklit, 2013). In our sample, kappa for inter-rater reliability on ASD diagnosis was 0.69 .

Peritraumatic dissociation was measured with the Peritraumatic Dissociative Experiences Questionnaire (Marmar et al., 1994), the most common measure to assess PD (van der Velden \& Wittmann, 2008). The 10-item self-report instrument assesses retrospective reports of derealization, depersonalization, and dissociative amnesia, out of body experiences and altered time perception or body image. Peritraumatic dissociative experiences are assessed on a Likert scale (1: not at all true, 5: extremely true). A cut-off score of 15 and higher can be used to identify highly dissociative individuals (Civilotti et al., 2015). The validated French version was used (Martin \& Marchand, 2003) which has good internal consistency (0.85) and test-retest reliability (0.88).

Peritraumatic distress was measured using the Peritraumatic Distress Inventory (Brunet et al., 2001). Thirteen items are answered on a four-point Likert scale (0; not at all, 4; extremely true) capturing experiences of perception of life threat (6 items) and negative emotions ( 7 items), during or immediately after the event. The internal consistency (0.83) and test-retest reliability (0.79) for the French version are good (Jehel, Brunet, Paterniti, \& Guelfi, 2005). A cut-off score of 14 and higher can be used to identify highly distressed individuals (Guardia et al., 2013).

Occurrence of stressful events and the availability of social support were measured respectively with the Social Readjustment Rating Scale (Holmes \& Rahe, 1967) and the Social Provisions Scale (Cutrona \& Russell, 1987). Occurrence of stressful events was scored with the sum of acute stressors identified by each participant, from a list of 43 stressing events (i.e., score of 1 to 43). The Social Provisions scale assessed the perceived availability of social support with 24 items. Internal consistency was excellent (0.96) in the French validation study (Caron, 1996). 
Exposure to past potentially traumatic events was measured by the Life Events Checklist, a widely used self-report measure, concurrently developed with the Clinician-Administered PTSD scale (Blake, Weathers, \& Nagy, 1998). Seventeen items assess exposure to potentially traumatic events using a Likert scale (it happened to me, I witnessed it, I have heard of it, n/a). Psychometric properties are adequate with fair reliability (stable over a 1-week period) and strong convergence with other measures associated with trauma exposures (e.g., Modified PTSD Symptom Scale)(Gray, Litz, Hsu, Lombardo, 2004).

\section{Data Analyses}

214 victims of violent crimes participated in this project. However, analyses were based on 162 participants (97 women) for whom complete data were available for the ASD. Our sample was distributed normally on main variables and covariates; therefore no data transformations were required.

Descriptive analyses were first performed using mean scores and standard deviations (SD). Potential covariates were included based on a review of significant ASD and PTSD risk factors (Armour \& Hansen, 2015; Brewin, Andrews, \& Valentine, 2000a; Hansen \& Elklit, 2011; Ozer et al., 2003) or were found to be significant (Pearson's $r>0.25$ ) risk factors for our population. They included number of potentially past traumatic events, previous PTSD diagnosis, perceived social support, additional stress following exposure to the event and type of event (e.g., sexual assault, physical assault). Preliminary analyses confirmed that previous potentially traumatic events appeared to be a significant moderator of the number of ASD symptoms and was therefore included in the final regression model below.

To meet the first objective, statistical analyses primarily involved multiple t-tests for men and women separately. T-tests were performed on overall scores for the acute stress 
reactions separately and then on scores from the PDI subscales (perception of life threat and negative emotions). As for the main analyses, a multiple hierarchical regression model was used to test whether peritraumatic dissociation (PD) and peritraumatic distress (PDI) predict ASD differentially according to gender. This model included 124 participants ( 75 women) because of missing data in some of the covariates. Predictor variables were standardized. ASD was entered as a continuous variable. Relevant covariates were entered simultaneously in Block 1; the main effects (PD and PDI) were entered in Block 2. Step 3, included the 2-way interaction terms (gender*dissociation, gender*distress, gender*previous traumatic events) and the 3-way interaction term (gender*distress*previous trauma) was entered in Block 4. Statistical significance was set at $p<0.05$. Significant interactions were evaluated using simple slopes analyses with lower and higher estimates for PDI and past potentially traumatic events based on values \pm 1 SD from the mean (Preacher, Curran, \& Bauer, 2006).

\section{Results}

Participant's characteristics are presented in Table 1. This study involved survivors of different types of crime, categorized as follows: a) physical assaults b) sexual assaults and/or domestic violence c) other violent crime which included receiving threats, home invasions, armed robbery or witnessing a violent crime. In our sample, $47.7 \%$ men $(n=31)$ and $53.6 \%$ women ( $\mathrm{n}=52)$ suffered from ASD (according to the number of symptoms met). Preliminary analyses revealed a gender difference according to the type of traumatic event $\left(\chi^{2}(2)=21.570, p\right.$ $<.001$ ), with more women victims of sexual assault or domestic violence compare to men. For men, physical assaults were more prevalent in our sample (see Table 1). Dummy variables were created on current trauma (type of crime) and then added as covariates in the hierarchical regression analysis. 


\section{Gender differences in peritraumatic dissociation and distress}

Women experienced significantly greater PDI $(t(157)=5.844, p=.005)$, more negative emotions $(t(158)=2.205 p=.001)$, and perceived life threat $(t(158)=0.630, p=.010)$, compared to men (see Table 1). No gender differences were found for peritraumatic dissociation.

\section{Identifying potential covariates}

Correlational analyses were first performed on the overall sample revealed significant moderate to high associations between peritraumatic variables and ASD. T-tests also revealed that individuals with a past PTSD diagnosis had a significantly higher number of ASD symptoms. Please refer to Table 2 for complete results. Associations between covariates and ASD (\# symptoms) were then analyzed for men and women separately and revealed somewhat different results with significant but modest associations with previous PTSD diagnosis as well as with previous potentially traumatic events in women but not men (see Table 2).

\section{Predictors of the number of ASD symptoms in men and women}

The regression model and reporting of standardized Betas $(\beta)$ are detailed in Table 3 . The simple slopes analysis below, however, gives the unstandardized coefficient $(b)$, as provided by analyses conducted on http://quantpsy.org/interact/mlr2.htm. Block 1 revealed that a previous PTSD significantly predicted current ASD symptoms. Greater peritraumatic dissociation and distress were both associated with a greater number of current ASD symptoms in Block 2 (see Table 3).

The 3-way interaction was statistically significant $(\beta=-0.312, t=-4.082, p<$ 0.001), and further analyses were conducted on this interaction term, as displayed in Figure 1 (men) and Figure 2 (women). Figure 1 indicates that greater levels of PDI and a high or average number of previous potential trauma experiences were associated with more ASD symptoms 
among men $(b=3.781, p<.001)$ and $(b=2.169, p<.001)$, respectively. PDI was not significantly associated with ASD risk in men who experienced a low number of past potentially traumatic experiences $(b=0.56, p=0.40)$. In women, high PDI is associated with more ASD symptoms even when they experienced few past potentially traumatic experiences $(b=1.39, p=0.02)$, as shown in Figure 2. Moreover, in women with a high number of these difficult past experiences, risk for ASD did not differ according to levels of peritraumatic distress $(b=-0.40, p=0.54)$. No significant multicollinearity was observed condition index $<3, \mathrm{VIF}<2$ for all variables (Belsely, Kuh, \& Welsch, 1980).

Two covariates included in the model (additional life stress and perceived social support) had more than $5 \%$ missing values. To verify the impact of missing data, we conducted a multiple imputation command. Regression results yielded from imputed datasets did not differ from the original data and as such, the latter were used for analyses $(\mathrm{N}=124)$.

\section{Post-hoc analyses}

To verify if our results could be explained by the fact that few women in our sample had low levels of PDI and that few men were found in the groups of high past potentially traumatic events and low distress, we conducted an additional mediation analysis. We examined whether peritraumatic distress mediated the relationship of past potentially traumatic events and number of ASD symptoms, and whether this differed for men and women. Covariates were as described previously. There was a significant relation between past potentially traumatic events and ASD $(b=0.333, p=0.001)$ in women. When PDI was controlled for, ASD was not predicted by previous potential traumas $(b=0.805, p=0.056)$, suggesting a partial mediation through PDI (Baron \& Kenny, 1986). The Sobel test confirmed an indirect effect of previous potential 
traumas through peritraumatic distress in women $(z=2.08, p=0.037)$. Among men, past potentially traumatic events did not predict PDI $(p=0.313)$.

\section{Discussion}

The current study investigated gender differences in peritraumatic reactions and ASD among VVCs. Results confirmed that peritraumatic reactions were significant predictors of ASD symptoms in both men and women, and found a gender-specific impact of past potentially traumatic events on the relation between peritraumatic distress and ASD symptoms. To our knowledge, this study is the first to show men's increased vulnerability for ASD as a result of cumulative effects of past potential traumatic experiences and PDI. In women, past potentially traumatic events increased risk for ASD as a result of experiencing higher PDI.

\section{Peritraumatic reactions in men and women}

As expected, high levels of acute stress reactions were observed in the current sample, and women reported significantly more negative emotions and higher perception of life threat compared to men. These findings are consistent with recent studies (Christiansen \& Hansen, 2015; Valdez \& Lilly, 2014; Johansen et al., 2006;). Display of emotional reactions to traumatic events, such as fear (also part of the A2 criteria), depend largely upon the subject's appraisal of the trauma. Greater PDI in our women participants may indicate their proneness to negatively assess the event compared to men, as previously suggested (Olff et al., 2007). Likewise, women also seem to be quicker in responding to threat signals. Alternatively, it has been stated that men usually show a greater sense of control (Olff et al., 2007) possibly preventing intense emotions to be registered. 
Unexpectedly, no gender differences were found for peritraumatic dissociation. These results are surprising since many studies seem to identify women as more vulnerable to PD (Ditlevsen \& Elklit, 2012; Lilly \& Valdez, 2012; Tolin \& Foa, 2006). Our results may reflect the propensity to endorse dissociative reactions demonstrated by our victims. Effectively, high levels of $\mathrm{PD}\left(\mathrm{M}_{\mathrm{PD}}=29\right)$ were experienced by both men and women, and the highly prevalent nature of dissociative symptoms around the time of the trauma could have limited variance and predictive power of this variable (Cardeña \& Carlson 2011). Furthermore, a recent study showed an increase in dissociative symptoms among females who reported more childhood and adulthood sexual trauma (Armour, Elklit, Lauterbach, \& Elhai, 2014). Differences in results might be explained by the fact that only one quarter of our female participants experienced a sexual aggression as the event of reference.

Peritraumatic reactions predicted a higher number of ASD symptoms in both men and women. Our main effect for PD might be explained by the ASD prevalence in our sample (47.7\% men and 53.6\% women suffered from ASD) compared to other studies with lower ASD rates. However, peritraumatic reactions did not predict the number of ASD symptoms differently for men and women. That gender did not moderate the relation between peritraumatic dissociation and ASD may suggest that our observed main effect for PD could be confounded by existing mental health issues understood as specific ASD responses, during the first days or weeks post-event (Keane, Kaufman, \& Kimble, 2004). Although we controlled for many variables identified in the literature, existing psychological problems were not taken into account in our regression model as they did not appear to be significant risk factors (e.g., Ozer et al., 2003). Similarly, Armour \& Hansen (2015) assessed subtypes of dissociation in ASD and found 
that being female and having a high number of reported traumas were not associated to the likelihood of having a more dissociative subtype.

\section{Number of past potentially traumatic events as a moderating variable of the relation between peritraumatic distress and ASD in men}

The number of past potentially traumatic events played a different role for men and women in predicting ASD, adding to the small body of research regarding gender differences in crime victim populations. More specifically, it moderated the relationship of PDI with ASD in men. The cumulative impact of multiple traumas and current distress may render them more vulnerable to ASD development. Our findings support the view that childhood and other previous traumas may confound the prediction of ASD (Bryant et al., 2011). A study identified young boys with past traumas as more vulnerable for adverse brain development (De Bellis \& Keshavan, 2003). It should be noted, moreover, that more than three quarters of our male participants were victims of physical assaults compared to $49 \%$ of women. This type of crime may have more serious consequences in combination to other past potentially traumatic events for men. Moreover, men often need to show they are "though" and in control (Levant, Rankin, Williams, Hasan \& Smalley, 2010) and admitting to have experienced distress during a physical assault could threaten this view. In contrast, having been exposed to few past potentially traumatic events for women was associated with greater ASD only in those with high distress. To our knowledge this has not been identified previously, as revictimization was related to aggravated ASD symptoms for both men and women in one study (Elklit, 2002).

\section{Peritraumatic distress as a mediator of the relation between the number of past potential traumatic events and ASD in women \\ Peritraumatic distress and past potentially traumatic events showed robust associations} with number of ASD symptoms in women, independently of socio-demographic characteristics 
and other psychological factors. In this study, PDI mediated the relationship between past potential traumatic events and ASD in women. Previous potential traumas increased risk for ASD symptoms in women by virtue of the greater peritraumatic distress it triggered. A greater number of past potentially traumatic events may heighten awareness to danger and could bring harsh negative emotions back to memory therefore producing, higher distress for women. The fact that this was not observed in men may come from men's tendency to experience emotions with lesser intensity (Grossman \& Wood, 2003). One previous study reported similar results with PTSD. Peritraumatic fear, horror and helplessness included in the PDI construct were among the few significant mediators to add a unique contribution to the relationship between gender and PTSD (Christiansen \& Hansen, 2015).

Our results endorse the importance of considering peritraumatic reactions and subjective experiences in understanding gender differences in ASD and give weight to the role of pre trauma factors in this relationship.

\section{Strengths, Limitations, and Implications}

This study contains a number of limitations. Some challenges inherent to VVC can partly explain study limitations such as varied antecedents of previous trauma. Legal procedures, also specific to crime victims, can impact their involvement in a study (Parsons \& Bergin, 2010). This could account for our moderate sample size (15\% of individuals did not fully complete the array of self-reports questionnaires). However, our socio-demographic data are comparable to studies with larger samples that tested sex/gender differences (Andrews, Brewin, \& Rose, 2003; Christiansen \& Hansen, 2015). Again, assessment of ASD diagnosis within 30 days was only made possible for $69 \%$ of participants. Although we believe this would not impact the results considering analyses were correlational in nature and were carried on continuous measures. This 
limitation is also present in several other studies (Elklit \& Brink, 2004; Elklit \& Christiansen, 2010; Hansen\& Elklit, 2013). Finally, the current investigation focused on men and women victims of violent crimes in Canada. Caution is advised for generalizability of results to all survivors of traumatic events, and cultural differences could apply. Further, most participants had a high number of ASD symptoms suggesting a possible selection bias. Victims with less severe symptoms of distress may have deemed intervention unnecessary, and thus decided not to participate in the study.

Nonetheless, the present investigation has numerous strengths and contributes to the literature in substantial ways. First, this is a prospective investigation and analyses performed were conservative in that they controlled for numerous confounding variables. Notably, this is the first study to directly test gender differences in peritraumatic variables' capacity to predict ASD symptoms among victims of violent crimes. Particularly novel was the exploration and weight given to past potentially traumatic events in explaining gender differences, as suggested in previous research (Crevier, Marchand, Nachar, \& Guay, 2013). To our knowledge, few studies regarding gender differences have used a semi-structured clinical interview to assess ASD (Armour et al., 2014). Typically, self-reported measures are used (Valdez \& Lilly, 2014), and ASD symptoms can be hard for victims to understand possibly weakening the specificity of the instrument. Indeed, many of our participants met criteria for ASD diagnosis in this study; in comparison with other studies on crime victims (Armour \& Hansen, 2015). Finally, acute stress reactions were tested with validated instruments instead of simply adding questions to other measures.

In sum, peritraumatic distress is an important peritraumatic risk factor for men and women. Recent studies did reach similar conclusions concerning the utility of A2 in predicting 
ASD, despite its suppression in DSM-5 (Christiansen \& Hansen, 2015; Hansen \& Elklit, 2011). Novel findings concern how pre and peritraumatic variables can account for gender differences in ASD. Clinically, this research suggests the potential need to establish gender specific assessments and interventions. For example, knowing that men with a history of previous potentially traumatic experiences and who experienced considerable PDI over the current event will be at greater risk for ASD development will assist clinicians in addressing specific pretraumatic factors when assessing male participants. Another key implication lies with the detrimental effect PDI can have on women. This shows the importance of assisting women who have experienced previous trauma before any revictimization occurs in the hope of reducing their risk later on. Adding cognitive measures in assessments as well as bearing in mind the importance of assessing the victim's appraisals of trauma to development and occurrence of peritraumatic distress could guide early interventions. Finally, this research reinforces the importance of considering gender-specific appraisals, as this could be the object of early interventions. 


\section{References}

Andrews, B., Brewin, C., \& Rose, S. (2003). Gender, social support, and PTSD in victims of violent crime. Journal of Traumatic Stress, 16(4), 421-427.

Armour, C., Elklit, A., Lauterbach, D., \& Elhai, J. D. (2014). The DSM-5 dissociative-PTSD subtype: Can levels of depression, anxiety, hostility, and sleeping difficulties differentiate between dissociative-PTSD and PTSD in rape and sexual assault victims. Journal of Anxiety Disorders, 28(4), 418-426.

Armour, C., \& Hansen, M. (2015). Assessing DSM-5 latent subtypes of acute stress disorder dissociative or intrusive? Psychiatry Research, 225(3), 476-483.

American Psychiatric Association. (2000). Diagnostic criteria from DSM-IV-tr. American Psychiatric Pub.

American Psychiatric Association (2013). Diagnostic and Statistical Manual of Mental Disorders (DSM-5®). American Psychiatric Pub.

Baron, R. M., \& Kenny, D. A. (1986). The moderator-mediator variable distinction in social psychological research: Conceptual, strategic, and statistical considerations. Journal of Personality and Social Psychology, 51(6), 1173.

Belsely, D. A., Kuh, E., \& Welsch, R. E. (1980). Regression Diagnostics. John Wiley \& Sons, New York.

Blake, D. D., Weathers, F. W., \& Nagy, L. M. Kaloupek, D.G., Charney, D.S. \& Keane, T.M. (1998). Clinician-Administered PTSD Scale for DSM-IV. Boston: National Center for Posttraumatic Stress Disorder.

Breh, D. C., \& Seidler, G. H. (2007). Is peritraumatic dissociation a risk factor for PTSD? Journal of Trauma \& Dissociation, 8(1), 53-69. 
Breslau, N., Kessler, R. C., Chilcoat, H. D., Schultz, L. R., Davis, G. C., \& Andreski, P. (1998).

Trauma and posttraumatic stress disorder in the community: the 1996 Detroit Area Survey of Trauma. Archives of General Psychiatry, 55(7), 626-632.

Breslau, N., Chilcoat, H. D., Kessler, R. C., Peterson, E. L., \& Lucia, V. C. (1999). Vulnerability to assaultive violence: Further specification of the sex difference in post-traumatic stress disorder. Psychological Medicine, 29(04), 813-821.

Breslau, N. (2001). The epidemiology of posttraumatic stress disorder: what is the extent of the problem? The Journal of Clinical Psychiatry.

Brewin, Andrews, B., \& Valentine, J. D. (2000a). Meta-analysis of risk factors for posttraumatic stress disorder in trauma-exposed adults. Journal of Consulting and Clinical Psychology, $68(5), 748-766$.

Brewin, C. R., Andrews, B., \& Rose, S. (2000b). Fear, helplessness, and horror in posttraumatic stress disorder: Investigating DSM-IV Criterion A2 in victims of violent crime. Journal of Traumatic Stress, 13(3), 499-509.

Brunet, A., Weiss, D. S., Metzler, T. J., Best, S. R., Neylan, T. C., Rogers, C., \& Marmar, C.R. (2001). The Peritraumatic Distress Inventory: a proposed measure of PTSD criterion A2. American Journal of Psychiatry, 158(9), 1480-1485.

Bryant, R. A. (2006). Cognitive-behavioral therapy for acute stress disorder. In Follette, Victoria M. and Ruzek, J.I. (Eds), Cognitive-Behavioral Therapies for Trauma, (2nd ed.). Guilford Press.

Bryant, R. A. (2007). Does dissociation further our understanding of PTSD? Journal of Anxiety Disorders, 21(2), 183-191.

Bryant, R. A., Harvey, A. G., Dang, S. T., Sackville, T., \& Basten, C. (1998). Treatment of acute 
stress disorder: a comparison of cognitive-behavioral therapy and supportive counseling. Journal of Consulting and Clinical Psychology, 66(5), 862.

Bryant, R. A., \& Harvey, A. G. (2003). Gender differences in the relationship between acute stress disorder and posttraumatic stress disorder following motor vehicle accidents. Australian and New Zealand Journal of Psychiatry, 37(2), 226-229.

Bryant, R. A., Friedman, M. J., Spiegel, D., Ursano, R., \& Strain, J. (2011). A review of acute stress disorder in DSM-5. Focus: the Journal of Lifelong Learning in Psychiatry, 9(3), 335350.

Cardeña, E., \& Carlson, E. (2011). Acute stress disorder revisited. Annual Review of Clinical Psychology, 7, 245-267.

Caron, J. (1996). L'Échelle de provisions sociales: une validation québécoise. Santé mentale au Québec, 21(2), 158-180.

Christiansen, D. M., \& Hansen, M. (2015). Accounting for sex differences in PTSD: A multivariable mediation model. European Journal of Psychotraumatology, 6.

Creamer, M., Burgess, P., Buckingham, W., \& Pattison, P. (1993). Posttrauma reactions following a multiple shooting. In International handbook of traumatic stress syndromes (pp. 201-212). Springer US.

Crevier, M. G., Marchand, A., Nachar, N., \& Guay, S. (2013). Overt social support behaviors: associations with PTSD, concurrent depressive symptoms and gender. Psychological Trauma: Theory, Research, Practice, and Policy, 6(5), 519.

Cutrona, C. E., \& Russell, D. W. (1987). The provisions of social relationships and adaptation to stress. Advances in Personal Relationships, 1(1), 37-67.

De Bellis, M. D., \& Keshavan, M. S. (2003). Sex differences in brain maturation in maltreatment-related pediatric posttraumatic stress disorder. Neuroscience \& Biobehavioral 
Reviews, 27(1), 103-117.

Ditlevsen, D. N., \& Elklit, A. (2012). Gender, trauma type, and PTSD prevalence: a re-analysis of 18 Nordic convenience samples. Annals of General Psychiatry, 11(1), 1-6.

Ehlers, A., \& Clark, D. M. (2000). A cognitive model of posttraumatic stress disorder. Behavior Research and Therapy, 38(4), 319-345.

Elklit, A. (2002). Acute stress disorder in victims of robbery and victims of assault. Journal of Interpersonal Violence, 17(8), 872-887.

Elklit, A., \& Brink, O. (2004). Acute stress disorder as a predictor of post-traumatic stress disorder in physical assault victims. Journal of Interpersonal Violence, 19(6), 709-726.

Elklit, A., \& Christiansen, D. M. (2010). ASD and PTSD in rape victims. Journal of Interpersonal Violence.

Feeny, N. C., Zoellner, L. A., \& Foa, E. B. (2000). Anger, dissociation, and posttraumatic stress disorder among female assault victims. Journal of Traumatic Stress, 13(1), 89-100.

Friedman, M. J., Keane, T. M., \& Resick, P. A. (2007). Handbook of PTSD: Science and practice. Guilford Press.

Fullerton, C. S., Ursano, R. J., Epstein, R. S., Crowley, B., Vance, K., Kao, T. C., Dougall, A. \& Baum, A. (2001). Gender differences in posttraumatic stress disorder after motor vehicle accidents. American Journal of Psychiatry.

Gray, M. J., Litz, B. T., Hsu, J. L., \& Lombardo, T. W. (2004). Psychometric properties of the life events checklist. Assessment, 11(4), 330-341.

Grieger, T. A., Fullerton, C. S., \& Ursano, R. J. (2003). Posttraumatic stress disorder, alcohol use, and perceived safety after the terrorist attack on the Pentagon. Psychiatric Services, 54(10), 1380-1382. 
Grossman, M., \& Wood, W. (1993). Sex differences in intensity of emotional experience: a social role interpretation. Journal of personality and social psychology, 65(5), 1010.

Hansen, M., \& Elklit, A. (2011). Predictors of acute stress disorder in response to bank robbery. European Journal of Psychotraumatology, 2.

Hansen, M., \& Elklit, A. (2013). Does acute stress disorder predict posttraumatic stress disorder following bank robbery? Journal of Interpersonal Violence, 28(1), 25-44.

Harvey, A. G., \& Bryant, R. A. (1999). The relationship between acute stress disorder and posttraumatic stress disorder: A 2-year prospective evaluation. Journal of Consulting and Clinical Psychology, 67(6), 985.

Hetzel-Riggin, M. D., \& Roby, R. P. (2013). Trauma type and gender effects on PTSD, general distress, and peritraumatic dissociation. Journal of Loss and Trauma, 18(1), 41-53.

Holmes, T. H., \& Rahe, R. H. (1967). The social readjustment rating scale. Journal of Psychosomatic Research, 11(2), 213-218.

Irish, L. A., Fischer, B., Fallon, W., Spoonster, E., Sledjeski, E. M., \& Delahanty, D. L. (2011). Gender differences in PTSD symptoms: an exploration of peritraumatic mechanisms. Journal of Anxiety Disorders, 25(2), 209-216.

Jehel, L., Brunet, A., Paterniti, S., \& Guelfi, J. D. (2005). [Validation of the peritraumatic distress inventory's french translation]. Canadian Journal of Psychiatry. Revue canadienne de psychiatrie, 50(1), 67-71.

Johansen, V. A., Wahl, A. K., Eilertsen, D. E., Hanestad, B. R., \& Weisaeth, L. (2006). Acute psychological reactions in assault victims of non-domestic violence: Peritraumatic dissociation, post-traumatic stress disorder, anxiety and depression. Nordic Journal of Psychiatry, 60(6), 452-462. 
Keane, T. M., Kaufman, M. L., \& Kimble, M. O. (2004). Peritraumatic dissociative symptoms, acute stress disorder, and the development of posttraumatic stress disorder: causation, correlation or epiphenomena (Vol. 3074). Presented at the constraint databases and applications: first international symposium, CDB 2004, Paris, France, June 12-13, 2004, Proceedings, Springer Science \& Business Media.

Kessler, R. C. (2000). Posttraumatic stress disorder: the burden to the individual and to society. Journal of Clinical Psychiatry 61(suppl 5), 1-478.

Kimerling, R., Ouimette, P., \& Wolfe, J. (Eds.). (2002). Gender and PTSD. Guilford Press.

Levant, R. F., Rankin, T. J., Williams, C. M., Hasan, N. T., \& Smalley, K. B. (2010). Evaluation of the factor structure and construct validity of scores on the Male Role Norms InventoryRevised (MRNI-R). Psychology of Men \& Masculinity, 11(1), 25.

Lilly, M. M., \& Valdez, C. E. (2012). Interpersonal trauma and PTSD: The roles of gender and a lifespan perspective in predicting risk. Psychological Trauma-Theory Research Practice and Policy, 4(1), 140.

Marmar, C. R., Weiss, D. S., Schlenger, W. E., Fairbank, J. A., Jordan, B. K., Kulka, R. A., \& Hough, R. L. (1994). Peritraumatic dissociation and posttraumatic stress in male Vietnam theater veterans. American Journal of Psychiatry, 151(6), 902-907.

Marshall, G. N., \& Schell, T. L. (2002). Reappraising the link between peritraumatic dissociation and PTSD symptom severity: Evidence from a longitudinal study of community violence survivors.Journal of Abnormal Psychology,111(4), 626.

Martin, A., \& Marchand, A. (2003). Prediction of posttraumatic stress disorder: peritraumatic dissociation, negative emotions and physical anxiety among French-speaking university students.Journal of Trauma \& Dissociation,4(2),49-63. 
McKibben, J. B., Bresnick, M. G., Askay, S. A. W., \& Fauerbach, J. A. (2008). Acute stress disorder and posttraumatic stress disorder: a prospective study of prevalence, course, and predictors in a sample with major burn injuries. Journal of Burn Care \& Research, 29(1), $22-35$.

Norris, F. H., \& Kaniasty, K. (1994). Psychological distress following criminal victimization in the general population: Cross-sectional, longitudinal, and prospective analyses. Journal of Consulting and Clinical Psychology, 62(1), 111.

Olff, M., Langeland, W., Draijer, N., \& Gersons, B. P. R. (2007). Gender differences in posttraumatic stress disorder. Psychological Bulletin, 133(2), 183-204.

Ozer, E.J., Best, S.R., Lipsey, T.L., \& Weiss, D.S. (2003). Predictors of posttraumatic stress disorder and symptoms in adults: a meta-analysis. Psychological Bulletin, 129 (1), 52-73.

Panasetis, P., \& Bryant, R. A. (2003). Peritraumatic versus persistent dissociation in acute stress disorder. Journal of Traumatic Stress, 16(6), 563-566.

Parsons, J., \& Bergin, T. (2010). The impact of criminal justice involvement on victims' mental health. Journal of Traumatic Stress, 23(2), 182-188.

Perry, B. D., Pollard, R. A., Blakley, T. L., Baker, W. L., \& Vigilante, D. (1995). Childhood trauma, the neurobiology of adaptation, and "Use dependent" Development of the brain: How "states" become "traits". Infant Mental Health Journal, 16(4), 271-291.

Preacher, K. J., Curran, P. J., \& Bauer, D. J. (2006). Computational tools for probing interactions in multiple linear regression, multilevel modeling, and latent curve analysis.Journal of Educational and Behavioral Statistics, 31(4), 437-448.

Stein, M. B., Walker, J. R., Hazen, A. L., \& Forde, D. R. (1997). Full and partial posttraumatic stress disorder: findings from a community survey. American Journal of Psychiatry, 154(8), 
$1114-1119$.

Steuwe, C., Lanius, R. A., \& Frewen, P. A. (2012). Evidence for a dissociative subtype of PTSD by latent profile and confirmatory factor analyses in a civilian sample. Depression and Anxiety, 29(8), 689-700.

Tolin, D. F., \& Foa, E. B. (2006). Sex differences in trauma and posttraumatic stress disorder: a quantitative review of 25 years of research. Psychological Bulletin, 132(6), 959-992.

Valdez, C. E., \& Lilly, M. M. (2014). Biological sex, gender role, and Criterion A2: Rethinking the "gender" gap in PTSD. Psychological Trauma-Theory Research Practice and Policy, $6(1), 34$.

Van Ameringen, M., Mancini, C., Patterson, B., \& Boyle, M. H. (2008). Post-traumatic stress disorder in Canada. CNS Neuroscience \& Therapeutics, 14(3), 171-181.

van der Velden, P. G., \& Wittmann, L.(2008).The independent predictive value of peritraumatic dissociation for PTSD symptomatology after type I trauma: a systematic review of prospective studies.Clinical Psychology Review,28(6),1009-1020. 
Table 1. Profile of the Participants and Gender Differences in Potential Covariates

\begin{tabular}{|c|c|c|}
\hline & $\operatorname{Men}(n=65)$ & Women $(n=97)$ \\
\hline Age (years $\pm \mathrm{SD})$ & $42(15.7)$ & $38(12.8)$ \\
\hline Marital Status n (\%) & & \\
\hline Single & 55.4 & 50 \\
\hline Married/Living with someone & 33.8 & 31.3 \\
\hline Separated/Divorced/Widowed & 10.8 & 18.8 \\
\hline Education $\mathrm{n}(\%)$ & & \\
\hline High School diploma or less & 60 & 59.8 \\
\hline Post-Secondary & 40 & 40.2 \\
\hline Employment Status (\%) & & \\
\hline Employed & 66.2 & 66 \\
\hline Previous PTSD (\%) & 13.8 & 18.6 \\
\hline Current traumatic Event $\mathrm{n}(\%)^{*}$ & & \\
\hline Physical Assault & 83.1 & 49.5 \\
\hline Sexual Assault or domestic violence & 3.1 & 26.8 \\
\hline Other violent crime (threats, witness) & 13.8 & 23.7 \\
\hline Peritraumatic Dissociation & 29.23 & 29.18 \\
\hline Peritraumatic Distress * & 25.71 & 30.84 \\
\hline Perceived life threat $*$ & 12.94 & 15.25 \\
\hline Negative emotions $* *$ & 12.92 & 15.59 \\
\hline ASD Diagnosis (\%) & 47.7 & 53.6 \\
\hline ASD symptoms (\#) & $12.12(4.07)$ & $13.13(3.54)$ \\
\hline
\end{tabular}

$* p=0.05, * * p=0.001$ 
Table 2. Univariate Associations (and p values) between ASD and Demographic variables, Peritraumatic Reactions, Potential covariates (Pearson's $r$ and t-tests) According to Gender

\begin{tabular}{|c|c|c|c|}
\hline & N Total & $\operatorname{Men}(n=65)$ & Women $(n=97)$ \\
\hline Age & ns & ns & ns \\
\hline Education & ns & ns & ${ }^{\mathrm{a}} 1.907(.035)$ \\
\hline Peritraumatic Dissociation & ${ }^{\mathrm{b}} .467(<.001)$ & ${ }^{\mathrm{b}} .528(<.001)$ & b $.426(<.001)$ \\
\hline Peritraumatic Distress & ${ }^{\mathrm{b}} .468(<.001)$ & b $.523(<.001)$ & b. $.388(<.001)$ \\
\hline Past PTSD Diagnosis & ${ }^{\mathrm{a}} 5.024(<.001)$ & ns & ${ }^{\mathrm{a}} 6.227(<.001)$ \\
\hline $\begin{array}{l}\text { Previous Potential Traumatic } \\
\text { Events }\end{array}$ & b $.177(.026)$ & ns & b $.270(.008)$ \\
\hline Additional Stress & ns & ns & ns \\
\hline Perceived Social Support & ns & ns & ns \\
\hline
\end{tabular}

${ }^{\mathrm{a}}: \mathrm{F}$ obtained from a $\mathrm{t}$ test

b: Pearson's correlation coefficient 
Table 3. Summary of Hierarchical Regression Model of Peritraumatic Dissociation and Distress in Predicting Acute Stress Disorder (\# symptoms) in Men and Women

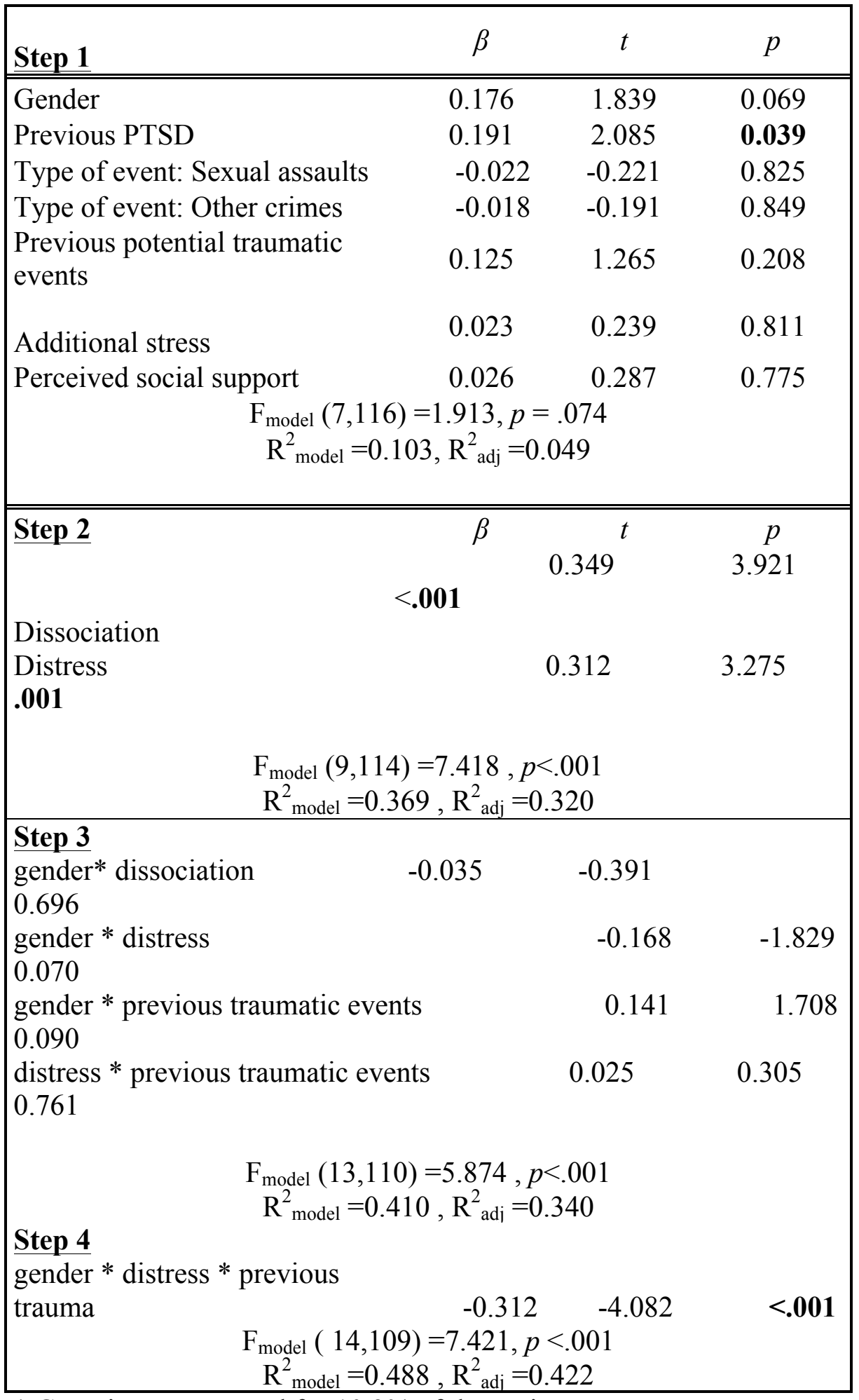

* Covariates accounted for $10.3 \%$ of the variance 


\section{Figure 1}

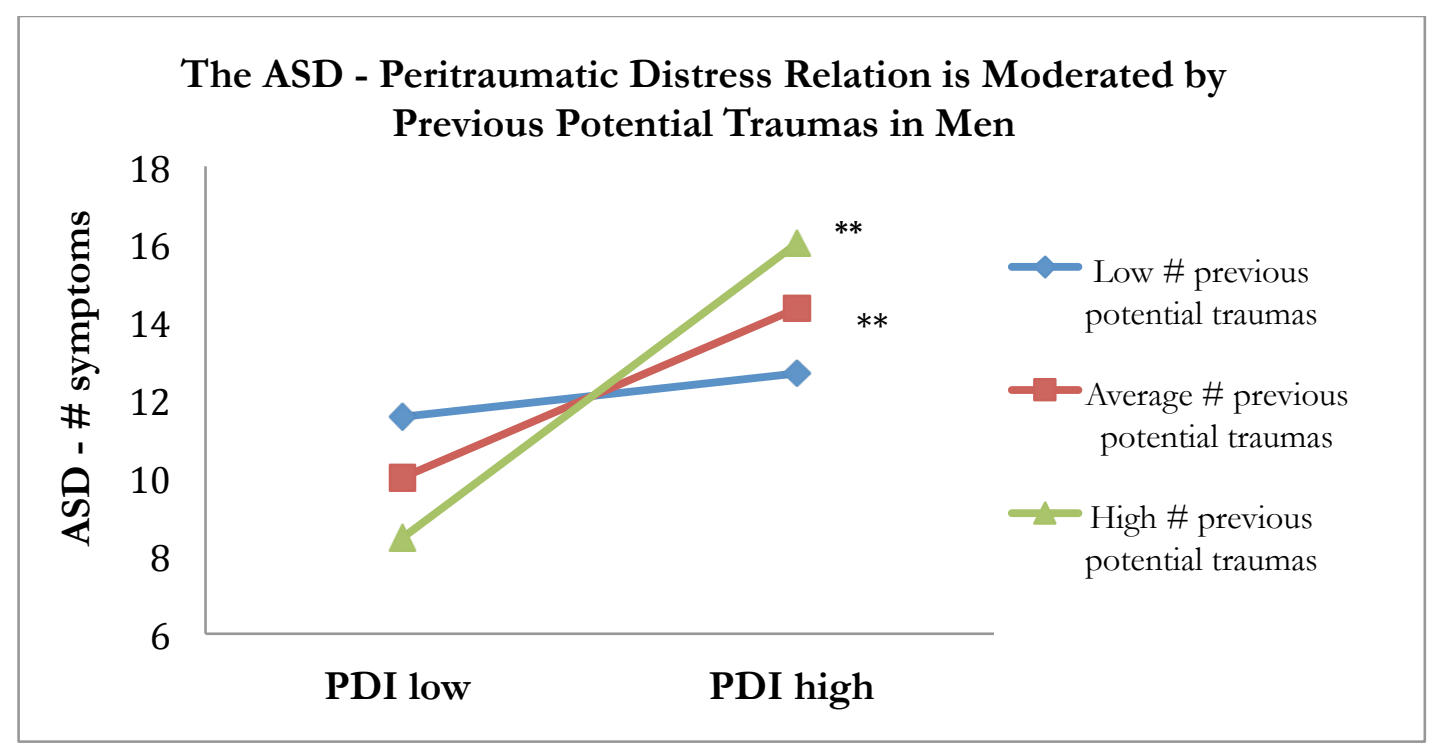

Figure 1: $(* *)$ Among men, experiencing high peritraumatic distress and moderate to high number of past potentially traumatic events are associated with a greater number of ASD symptoms $(b=3.78$ and $b=2.17$, respectively $p<0.001)$. PDI and number of past potentially traumatic events are depicted as Mean \pm SD. 
Figure 2

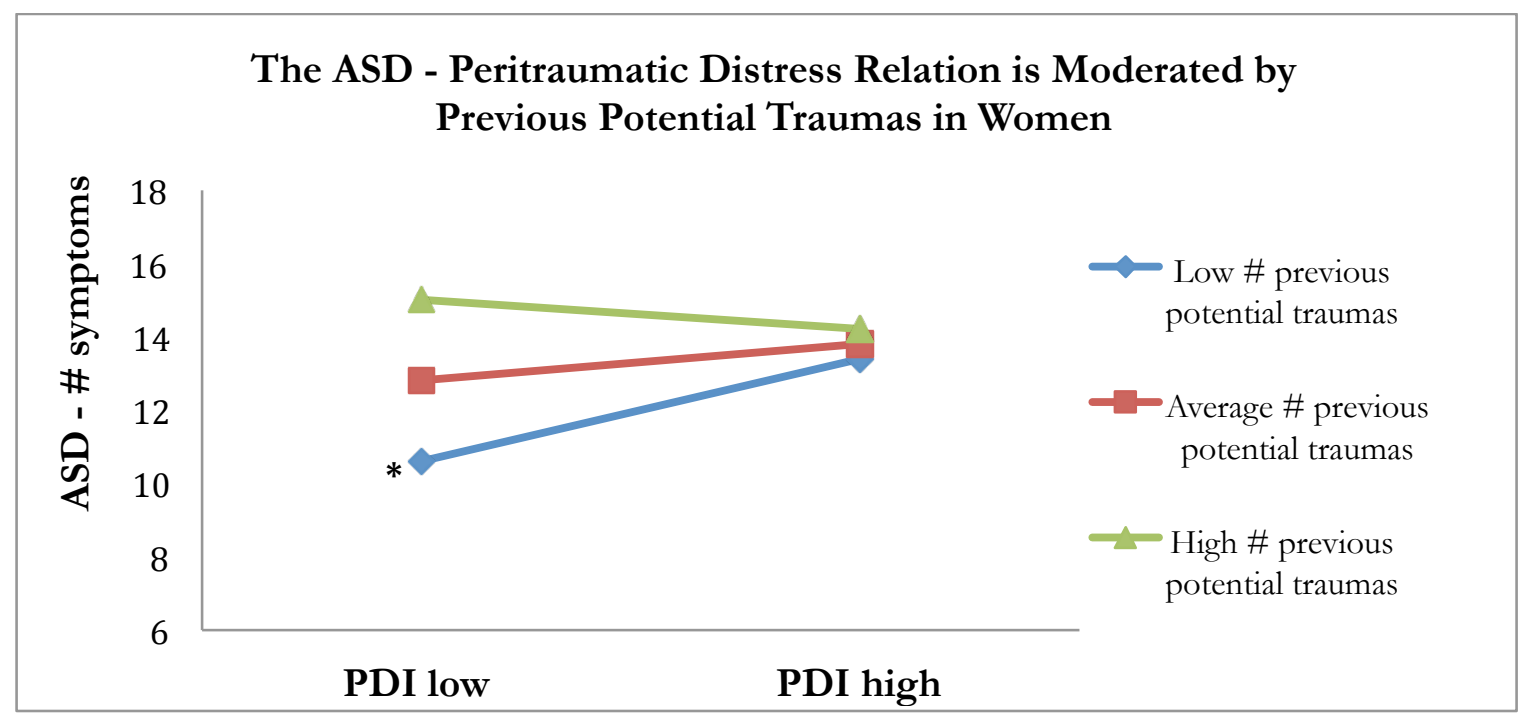

Figure 2: $(* b=1.39, p=0.02)$. PDI and number of past potentially traumatic events are depicted as Mean \pm 1 SD. 